\title{
SOME RESULTS ON TOPOLOGICAL NEIGHBOURHOODS
}

\author{
BY C. P. ROURKE AND B. J. SANDERSON
}

Communicated by William Browder, December 5, 1969

Full proofs of results announced here are to be found in [10]. We consider the problem of classifying germs of neighbourhoods of locally flat topological submanifolds. Let $M^{n}$ be a fixed topological manifold. An $r$-neighbourhood of $M$ is a pair $(i, N)$ where $N$ is a topological manifold of dimension $n+r$ and $i: M \rightarrow N$ a locally flat embedding satisfying $i^{-1}(\partial N)=\partial M$. Denote the set of germs of $r$-neighbourhoods (i.e. equivalence classes under homeomorphism defined near $i(M)$ and fixing $M$ ) by $\mathfrak{N}_{r}(M)$.

MAIN THEOREM. There is a countable CW complex $\mathrm{B} \mathrm{Top}_{r}$ and a function $c: \Re_{r}(M) \rightarrow[M, B$ Top $r]$, which is a bijection if $\partial M=\varnothing$ and $(n, r) \neq(1,3)$, or if $\partial M \neq \varnothing$ and $(n, r) \neq(1,3)$ or $(2,3)$.

The omitted cases in the theorem are due to the unsolved 4-dimensional annulus conjecture; an extension to any one of these cases is strictly equivalent to the conjecture.

OUTLINE OF PROOF. We rework Haefliger's theory of microbundle pairs [2] in the topological category. The crucial tool is immersion theory. An r-microbundle pair is a pair $\epsilon^{k} C \zeta^{k+r}$ of microbundles, where $\epsilon^{k}$ denotes the trivial bundle of rank $k$ and the inclusion is locally trivial. Two such are equivalent (stably) if they are isomorphic after possibly adding further trivial bundles to both elements and the isomorphism is the identity on the trivial sub-bundle. The set of equivalence classes forms a good 'theory'; the group is Top r $=\lim _{k \rightarrow \infty}\left\{\operatorname{Top}_{r+k, k}\right\}$, where Top $r+k, k=$ germs of automorphisms of $R^{r+k}$ fixing $\left.\{0\} \times R^{k}\right\}$; the classifying space is $B \operatorname{Top}_{r}$. We associate to the neighbourhood $(i, N)$ the pair $\tau_{M} \oplus \nu \subset i^{*}\left(\tau_{N}\right) \oplus \nu$, where $\nu$ is a stable inverse to $\tau_{M}$ and this defines the function $c$. Using immersion theory we show that a germ of neighbourhood is equivalent to a pair $\tau_{M} \subset \xi^{n+r}$ and then the theorem follows by diagram chasing from:

FIRST STABILITY THEOREM. $\pi_{i}\left(\right.$ Top $\left._{r+k, k}\right) \rightarrow \pi_{i}\left(\right.$ Top $\left._{r}\right)$ is an isomorphism provided $i \leqq k$ and either $k+r \geqq 5$ or $r \leqq 2$.

This follows from the PL result (Haefliger [2], see also [11, III 5.4]) and the analogous result for $\mathrm{Top}_{r+k, k} / \mathrm{PL}_{r+k, k}$ which, using

AMS 1969 subject classifications. Primary 5701, 5705; Secondary 5730, 5478, 5560.

Key words and phrases. Classification of germs of topological neighborhoods of locally flat submanifolds, microbundle pairs, normal block bundles, normal bundles, approximation and triangulation. 
immersion theory again, reduces to a statement about straightening handles (in the sense of Kirby-Siebenmann [7]) keeping a PL subhandle fixed. For $r \leqq 2$ this follows from the results of Kirby [5] and for $k+r \geqq 5$ we follow the methods of [7] appealing to the relative surgery techniques of [9]. The relative statement of immersion theory that we need is contained in an appendix to [10] written by Armstrong and the first author and follows from the relative local contractibility theorem of Edwards and Kirby [6].

Applications.

1. Normal block bundles. Let Top $\tilde{r}$, the analogue of $\mathrm{PL}_{r}^{\sim}[11]$, be the group for (closed) topological block bundles. Suppose $M^{n} \subset Q^{n+r}$ is a locally flat topological pair with $\partial Q \cap M=\partial M$ and that $|K|=M$ is a triangulation (not assumed to be combinational). There is a map $\chi: B \operatorname{Top}_{r} \rightarrow B$ Top $r$ and, in the dimensions of the main theorem, liftings of $c(\mathrm{id}, Q)$ over $\chi$ correspond to isotopy classes of normal block bundles $\xi^{r} / K$ on $M$ in $Q$ (in the sense of $[11, \mathrm{I} \$ 4]$ ). We next prove a stability theorem for $\mathrm{Top}_{r+k, k}^{\sim} / \mathrm{PL}_{r+k, k}^{\sim}$ by again reducing to relative handle straightening and deduce homotopy properties of $\chi$ using [11, III 2.1]. There is an analogous treatment of open and micro block bundles and we obtain:

THEOREM. There is a normal open, closed or micro block bundle $\xi^{r} / K$ on $M$ in $Q$ unique up to isotopy in the following cases:

(1) $r \geqq 5$ or $\leqq 2$,

(2) $r=4$ and both $M$ and $\partial M$ are 1-connected,

(3) $r=3$ and both $M$ and $\partial M$ are 2-connected.

2. Normal bundles. Let $M$ and $Q$ be as above and $M$ not necessarily triangulable. We have a similar analysis for normal open and closed disc and microbundles on $M$ in $Q$ (by Kister [8] the theories of open disc and microbundles coincide), and using Haefliger and Wall's results [3] for the $\mathrm{PL}$ case we obtain:

THEOREM. Suppose $r \geqq 5$; if $n \leqq r$ then $M$ has a normal microbundle in $Q$, if $n<r$ it is unique up to isotopy. Suppose $r \geqq 6$; if $n \leqq r-1$ then $M$ has a normal closed disc bundle in $Q$ and if $n<r-1$ it is unique up to isotopy.

3. Approximation and triangulation. Combining our results with the triangulation theorems of Kirby and Siebenmann [7] we obtain obstruction theories analogous to those of Hirsch [4] and Haefliger [1] for the problems of triangulating a locally flat submanifold of PL manifold and for the problem of approximating a locally flat embedding of PL manifolds by a PL embedding; the coefficients are in $\pi_{i}\left(\mathrm{Top}_{r} / \mathrm{PL}_{r}\right)$ and $\pi_{i}\left(\mathrm{Top} ; \mathrm{PL}, \mathrm{Top}_{r} ; \mathrm{PL}_{r}\right)$ respectively. But Top $\mathrm{p}_{r} / \mathrm{PL}_{r}$ 
is contractible if $r \leqq 2$ by Kirby [5] and Wall [12] while if $r \geqq 3$ we have:

SECond STABILITy THEOREM. The natural map Top $_{r} / \mathrm{PL}_{r} \rightarrow$ Top/PL is a homotopy equivalence if $r \geqq 3$.

We deduce:

Theorem. (1) Suppose $i: M^{n} \rightarrow Q^{n+r}$ is a locally flat embedding of PL manifolds and $i \mid \partial M$ is PL then if $r \geqq 3$ there is an ambient $\epsilon$-isotopy of $Q$ rel $\partial Q$ carrying $i$ to a $\mathrm{PL}$ embedding. The same is true if $r \leqq 2, n+r \geqq 5$ and an obstruction in $H^{3}\left(M, \partial M ; Z_{2}\right)$ vanishes.

(2) Suppose $i: M^{n} \rightarrow Q^{n+r}$ is a locally flat embedding and $Q$ is a $\mathrm{PL}$ manifold. Suppose $i(\partial M)$ is a PL submanifold of $\partial Q$ then if $n \geqq 5$ and $r \leqq 5$ there is an ambient $\epsilon$-isotopy of $Q$ rel $\partial Q$ carrying $M$ to a PL submanifold. If $r \geqq 3$ the same is true if and only if the given triangulation of $\partial M$ extends over $M$.

From the approximation theorem ${ }^{1}$ (part (1) of above) we have:

COROLlARY. The PL and locally flat knot problems coincide in codim $\geqq 3$ (i.e. there is a natural bijection between isotopy classes of PL and locally flat embeddings of one PL manifold in another).

\section{REFERENCES}

1. A. Haefliger, Report to the I.C.M., Moscow, 1966.

2. —_, Lissage des immersions. II (preprint).

3. A. Haefliger and C. T. C. Wall, Piecewise linear bundles in the stable range, Topology 4 (1965), 209-214, MR $32 \# 1716$.

4. M. Hirsch, Obstruction theories for smoothing manifolds and maps, Bull. Amer. Math. Soc. 69 (1963), 352-356. MR $26 \# 6980$.

5. R. C. Kirby, Locally flat submanifolds in codim 2, Proc. Topology Conference, Georgia 1969.

6. R. C. Kirby and R. Edwards, On deformations of spaces of embeddings, (to appear).

7. R. C. Kirby and L. Siebenmann, On the triangulation of manifolds and the Hauptvermutung, Bull. Amer. Math. Soc. 75 (1969), 742-749.

8. J. Kister, Microbundles are fibre bundles, Ann. of Math. (2) 80 (1964), 190-199. MR $31 \# 5216$.

9. C. P. Rourke, Block bundles and Sullivan theory, Compositio Math. (to appear).

10. C. P. Rourke and B. J. Sanderson, On topological neighbourhoods, Compositio Math. (to appear).

11. - Block bundles. I, II, III, Ann. of Math. (2) 87 (1968), 1-28, 256-278, 431-483. MR 37 \#2234a, b; MR $38 \# 729$.

12. C. T. C. Wall, PL, submanifolds with codimension two, Proc. Cambridge Philos. Soc. 63 (1967), 5-8. MR 37 \#3577.

Mathematics Institute, University of Warwick, Coventry, England

1 The approximation theorem is "well known." Originally announced by Bryant and Seebeck the result has also been proved by Miller. Rushing, Connelly and Cobb. 